\title{
The use of geometric effects in diagnosing ion density in ICF-related dot spectroscopy experiments
}

\author{
G. Pérez-Callejo ${ }^{\mathrm{a}, *}$, D. A. Liedahl ${ }^{\mathrm{b}}$, M. B. Schneider ${ }^{\mathrm{b}}$, S. J. Rose ${ }^{\mathrm{a}, \mathrm{c}}$, J. S. \\ Wark $^{\mathrm{a}}$ \\ ${ }^{a}$ Department of Physics, Clarendon Laboratory, University of Oxford, Parks Road, Oxford \\ $O X 13 P U, U K$ \\ ${ }^{b}$ Lawrence Livermore National Laboratory, Livermore, California 94550, USA \\ ${ }^{c}$ Plasma Physics Group, The Blackett Laboratory, Imperial College London, Prince Consort \\ Road, London, SW7 2AZ, UK
}

\begin{abstract}
We describe a method to calculate the ion density of High Energy Density (HED) cylindrical plasmas used in Dot Spectroscopy experiments. This method requires only spectroscopic measurements of the $\mathrm{He} \alpha$ region obtained from two views (Face-on and Side-on). We make use of the fact that the geometry of the plasma affects the observed flux of optically thick lines. The ion density can be derived from the aspect ratio (height-to-radius) of the cylinder and the optical depth of the He $\alpha-y$ line $\left(1 s 2 p{ }^{3} P_{1} \rightarrow 1 s^{2}{ }^{1} S_{0}\right)$. The aspect ratio and the optical depth of the $y$ line are obtained from the spectra using ratios measured from the two directions of emission of the optically thick He $\alpha$ - $w$ line $\left(1 s 2 p{ }^{1} P_{1} \rightarrow 1 s^{2}{ }^{1} S_{0}\right)$ and the ratio of the optically thick to thin lines. The method can be applied to mid-Z elements at ion densities of $10^{19}-10^{20} \mathrm{~cm}^{-3}$ and temperatures of a the order of keV, which is a relevant regime for Inertial Confinement Fusion (ICF) experiments.
\end{abstract}

Keywords: ion density, dot spectroscopy, cylindrical plasmas, x-ray spectroscopy

\footnotetext{
${ }^{*}$ Corresponding author: gabriel.perezcallejo@physics.ox.ac.uk
} 


\section{Introduction}

Measuring the temperature and density of plasmas in High Energy Density (HED) experiments is fundamental to understanding the conditions of the plasma and to design better experimental set-ups. Spectroscopy is a commonly used method for temperature [1, 2, 3, 4, 5, 6, 7, 8, 9, 10, 11] and plasma density measurements, with various different methods being applied depending on the temperatures and densities involved.

In the high density regime, such as the plasma within an Inertial Confinement Fusion (ICF) implosion core, one of the most common spectroscopic approaches for measuring the plasma density is the study of Stark broadening of spectral lines 12, 13, 3. However, for plasmas at lower densities, such as the plasma that fills a hohlraum in indirect drive ICF, the Stark broadening is smaller than thermal broadening associated with the Doppler effect. The line profile is then usually Gaussian or Voigt-like, and since the contribution from the Stark effect must be isolated [14], large uncertainties in the measurement are inevitable.

If diagnosis via Stark broadening is not appropriate, there are some other approaches to characterzing the plasma density using its continuum emission [15], fluorescence emission [16], or the study of line ratios. The relative intensity of lines can provide information about the atomic level population distribution in a plasma and therefore its density. In 1969, Gabriel and Jordan [17] developed a method for characterization of the solar corona that uses line ratios from the He $\alpha$ complex $\left(1 s 2 l \rightarrow 1 s^{2}\right)$. This complex is made up of four lines (which we name according to Gabriel's work [18]), namely the resonance or $w$ line $\left(1 s 2 p{ }^{1} P_{1} \rightarrow\right.$ $\left.1 s^{2}{ }^{1} S_{0}\right)$, two intercombination lines, the $x$ line $\left(1 s 2 p^{3} P_{2} \rightarrow 1 s^{2}{ }^{1} S_{0}\right)$ and the $y$ line $\left(1 s 2 p^{3} P_{1} \rightarrow 1 s^{2}{ }^{1} S_{0}\right)$; and the forbidden or $z$ line $\left(1 s 2 s^{3} S_{1} \rightarrow 1 s^{2}{ }^{1} S_{0}\right)$. In particular, their method uses the ratio of intensities $z /(x+y)$ to characterize the electron density of the plasma. Gabriel and Jordan's method has been widely used for different regions of the solar corona [19, 20, 21, 22] and given its great potential, there have also been many efforts to extend it to different astrophysical plasmas, such as planetary nebulae [23] and stellar winds and 
coronae $24,25,26,27$. However, this method is only valid at relatively low ion densities $\left(n_{i}<10^{12}-10^{13} \mathrm{~cm}^{-3}\right)$, as for higher densities the flux from the $z$ and $x$ lines diminishes to the point of being unobservable, and the $w$ and $y$ lines are the only remaining He-like components, usually blended with Li-like satellite emission.

The use of the ratio of these two remaining lines $w / y$ as a density diagnostic for laser-generated plasmas was first studied by Vinogradov et al. 28] and was further improved in the following years [29, 30, 31. After that, the $w / y$ ratio became a commonly used measurement for measuring the density of laserproduced plasmas [32, 33, 34, 35] and Tokamaks [36, 37]. Nevertheless, as the opacity of the $w$ line is $\sim 15$ times greater than that of the $y$ line for mid-Z elements, in HED conditions the $w$ line becomes optically thick whereas the $y$ line does not. Therefore, opacity effects must be taken into account when making diagnostic inferences based upon those lines.

While one might expect the presence of line opacity to strictly reduce the line flux relative to the optically thin limit, Bhatia and co-workers [38, 39, 40, showed that, under certain conditions, opacity can cause an relative enhancement of the emission of optically thick lines. The work of Kerr et al. [41, 42, 43] using the radiation transport code CRETIN [4], showed that this enhancement was related to the geometry of the plasma and the Line Of Sight (LOS) used to obtain the spectra. Therefore, for non-spherical plasmas, the enhancement of optically thick lines depends on the position of the observer with respect to the plasma. This effect has been observed in astrophysical plasmas [45] and has applications to charaterizing the geometry of astrophysical bodies from their line ratios 42, 46. However, to our knowledge, it has never been used for density measurements in laboratory plasmas (although a potential experiment to observe these effects was proposed by Mancini et al. [47]).

Over the past few years there has been an increasing interest in using dot spectroscopy [4, 5] for diagnosing the temperature in plasmas in ICF hohlraums [8, 9, 10, 11]. The dots are cylinders of mid-Z materials with a thickness of $\sim 0.1-0.3 \mu \mathrm{m}$ and a diameter of the order of $250-800 \mu \mathrm{m}$, as this geome- 
try is easy to manufacture. Recently, the dot spectroscopy method has been used to validate results from radiation hydrodynamics simulations [48, for ICF hohlraums at the National Ignition Facility [49] (NIF). We focus on the geometric effects in cylinders of mid-Z elements at ion densities $n_{i} \sim 10^{19}-10^{20} \mathrm{~cm}^{-3}$ and temperatures of the order of $\mathrm{keV}$. This is typical of the range of conditions in ICF experiments. The choice of mid-Z elements is motivated by the fact that their $\operatorname{He} \alpha$ and Ly $\alpha$ lines $(\sim 4-8 \mathrm{keV})$ lie outside the region of emission of the gold plasma inside the hohlraum $(\sim 3 \mathrm{keV})$. Therefore, the spectral background in the region of interest is usually negligible.

In the experiments described in Barrios et al. [10, 11, the dots were coated on top of the $\mathrm{CH}$ capsule or on a film inside the hohlraum. As the laser heats up the system, the $\mathrm{CH}$ capsule keeps the dots tamped and they expand in the axial and radial directions. Early in the expansion, the geometry of the dot remains cylindrical, given the symmetry of the system, although later in time its geometry might change, as the assumption of pure planar expansion starts to break down. We make use of the characteristic way in which the cylindrical geometry of the dots modifies their spectral pattern in the early stages of the expansion to diagnose its density.

We present a method for calculating the ion density of cylindrical plasmas making use of the effects of their geometry in their radiation pattern. This method requires two measurements of the He $\alpha$ spectrum (the spectral flux in the axial and the radial directions), one of which can be the already existent measurement in the dot spectroscopy method for temperature diagnostic. We shall assume that the plasma of study is cylindrical and completely uniform (single temperature and density) and constant in time. Given that for the conditions of study, Doppler broadening is approximately one order of magnitude greater than Stark broadening 1 [51, 52, 53] we also assume Gaussian lineshapes, but the method can be extended to different lineshapes, such as a Lorentzian

\footnotetext{
${ }^{1}$ This result was verified by the authors using the atomic kinetics code SCRAM [50] and CRETIN
} 
or a Voigt profile. We focus on the $w$ and $y$ components of the He $\alpha$ complex. In the conditions of study, it is usually the case that the $w$ line is significantly optically thick $\left(1-e^{-\tau_{w}} \sim 1\right.$ near line center) whereas the $y$ line is not. We note that for the method presented here, it is not necessary that the $y$ line is strictly optically thin $\left(\tau_{y}<1\right)$, but it can have optical depth of a few. We assume that these conditions hold for our case.

We consider for simplicity the case of a single element plasma. This is not a necessary assumption, as the method is still valid for a multi-species plasma as long as no lines from one element overlap with the He $\alpha$ complex of another. In the case of more than one element being present in the plasma, the He $\alpha$ spectrum of each element can be used to obtain the ion density of that particular element. The total ion density can then be calculated as the sum of the individual contributions, provided that the assumption of a uniform plasma holds.

The paper is organized as follows. First we discuss radiation transport in cylindrical plasmas. Then the expressions for the flux in two directions of emission are derived. We use these expressions to calculate the line ratios between the $w$ and $y$ lines for the axial and radial directions and show that they are functions of the optical depth and geometry of the plasma. We then express the ion density as a function of these variables. An example is given in section 5 to illustrate the method. Finally we discuss the limitations of this method when the geometry is not perfectly cylindrical.

\section{Theory for a plasma cylinder}

The radiation transfer equation in one dimension, for transfer in the direction of propagation, shows that the intensity increases from the plasma emissivity and decreases from its absorption [54]

$$
\left[c^{-1} \frac{\partial}{\partial t}+\frac{\partial}{\partial z}\right] I=\eta-\kappa I,
$$

where $c$ is the speed of light, $\eta$ represents the emission coefficient or emissivity of the system (with units of $\mathrm{erg} / \mathrm{Hz} / \mathrm{sr} / \mathrm{s} / \mathrm{cm}^{3}$ in the cgs system) and $\kappa$ its 


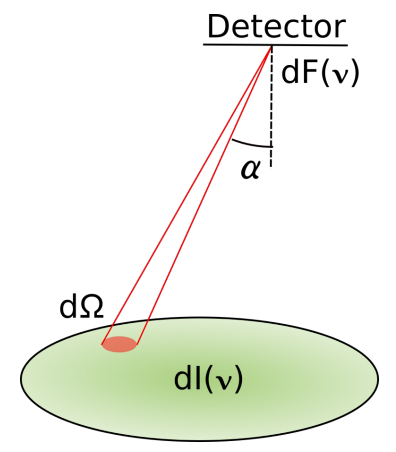

Figure 1: Differential element of spectral flux. As the detector is placed further from the plasma, the angle $\alpha$ becomes smaller, until all the rays reaching the detector are parallel and with $\alpha=0$.

absorption coefficient or opacity $\left(\mathrm{cm}^{-1}\right)$. I has units of $\mathrm{erg} / \mathrm{Hz} / \mathrm{sr} / \mathrm{s} / \mathrm{cm}^{2}$. We consider instead the time-independent version of this equation as one of our assumptions is that the conditions are constant and uniform. In experimental conditions, however, the plasma conditions are likely to evolve over time. In that case, it is still possible to neglect the time-dependent term as long as the temporal variations happen on a timescale $\Delta t$ such that $\Delta t \gg \Delta z / c$, where $\Delta z$ is the lengthscale of the plasma. As an example, for a plasma $100 \mu \mathrm{m}$ thick, it is possible to neglect temporal variations slower than $\sim 0.3 \mathrm{ps}$, while most ICF experiments evolve on the nanosecond scale. Dropping the time-dependence of equation 1, we obtain

$$
\frac{\partial}{\partial z} I=\eta-\kappa I
$$

If there is no external illumination on the plasma, equation 2 has the solution

$$
I(\nu, z)=\frac{\eta(\nu)}{\kappa(\nu)}\left(1-e^{-\kappa(\nu) z}\right)=S(\nu)\left(1-e^{-\kappa(\nu) z}\right)
$$

where $S(\nu)=\eta(\nu) / \kappa(\nu)$ is the source function for radiation of frequency $\nu$ and $z$ is the distance that the radiation travels through the plasma. The quantity $\kappa z$ is the optical depth.

For a single Gaussian-shaped line, the emissivity and opacity coefficients can 
be written as

$$
\begin{aligned}
& \eta(x)=\eta_{0} e^{-x^{2}}, \\
& \kappa(x)=\kappa_{0} e^{-x^{2}},
\end{aligned}
$$

where $\eta_{0}$ and $\kappa_{0}$ are respectively the emissivity and opacity at line center, and $x$ is the dimensionless frequency

$$
x=\frac{\nu-\nu_{0}}{\nu_{D}},
$$

with $\nu_{0}$ the frequency of the transition at line center and $\nu_{D}$ the Doppler width. Therefore, we can write the intensity of a particular line as

$$
I(x, z)=\frac{\eta(x)}{\kappa(x)}\left(1-e^{-\kappa(x) z}\right)=S_{0}\left(1-e^{-\kappa(x) z}\right),
$$

where $S_{0}$ is the source function at line center. For the sake of clarity, the explicit inclusion in the notation of an $x$-dependence is hereafter suppressed.

For practical applications it is usually more appropriate to work with the spectral flux, as that is the observable measured by a detector. It is given by

$$
F(\nu)=\int d \Omega I(\nu) \cos \alpha,
$$

where $d \Omega$ is the differential solid angle subtended by the source at the detector and $\alpha$ is defined as the angle between a ray leaving the source and the normal vector of the corresponding differential surface element at the detector (figure 11. $F$ has units of $\mathrm{erg} / \mathrm{Hz} / \mathrm{s} / \mathrm{cm}^{2}$.

To exploit the symmetries inherent in the cylindrical geometry, we choose to focus here on the axial flux, or Face-on; and the radial flux, or Side-on. These correspond to radiation leaving the cylinder normal and parallel to its base, respectively. Our model consists of a cylindrical plasma at which two detectors are aimed, one that measures Face-on flux, while the second measures the Sideon flux, as shown in figure 2 (although this process can be extended to different positions of the detectors). Both detectors are assumed to be sufficiently far from the target so that all the rays reaching them are parallel, thus allowing us to set $\alpha \sim 0$. The detectors are also assumed to cover the whole size of the plasma and to be at the same distance $d$ from the cylinder. 


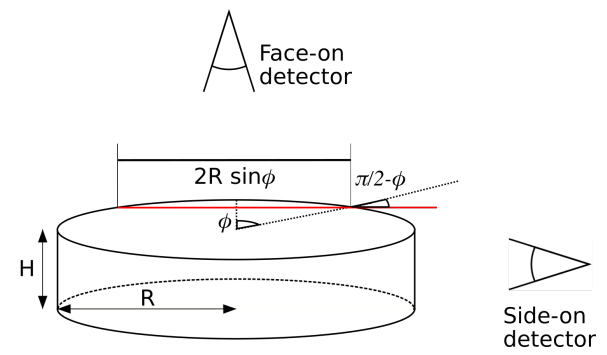

(a)

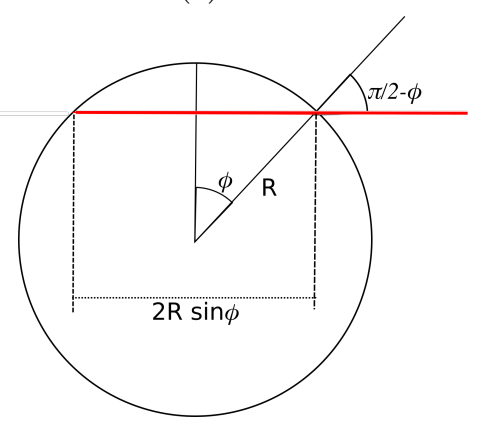

(b)

Figure 2: Schematic of the cylindrical plasma and the Face-on and Side-on detectors. 2a Shows the whole system and $2 \mathrm{~b}$ the top view only, showing one ray (red) travelling to the Side-on detector. For Face-on detection, all the photons travel the same distance $H$, and move perpendicularly to the emitting surface, in this case, the face of the cylinder. For Side-on view, rays span a range of chord lengths from 0 to $2 R$. If the azimuthal angle $\phi$ is defined as shown, a photon travels a distance $2 R \sin \phi$ before leaving the plasma, and exits with an angle $\pi / 2-\phi$ with respect to the surface normal vector.

\subsection{Face-on flux}

For radiation leaving the plasma in the axial direction equation 7 becomes

$$
I=S_{0}\left(1-e^{-\kappa H}\right),
$$

where $H$ is the height of the plasma cylinder. The radiation exits the plasma perpendicular to its surface. Therefore, from equation 8 , it follows

$$
F^{\text {face }}=\frac{\pi R^{2}}{d^{2}} S_{0}\left(1-e^{-\kappa H}\right)
$$

where $R$ is the radius of the cylinder, $\pi R^{2}$ is the emitting surface (the base of the cylinder) and $d$ is the distance from the detector to the plasma cylinder. 
From equation 10, the frequency-integrated flux of a given spectral line in the Face-on orientation is

$$
F_{\text {line }}^{\text {face }}=\frac{\pi R^{2}}{d^{2}} S_{0} \int_{-\infty}^{+\infty} d x\left(1-\exp \left[-\kappa_{0} H e^{-x^{2}}\right]\right) .
$$

To simplify the notation we define $G^{\text {face }}$ as the integral over frequencies for Face-on flux (which only depends on the product of $\kappa_{0}$ and $H$ ), that is

$$
G^{f a c e}=\int_{-\infty}^{+\infty} d x\left(1-\exp \left[-\kappa_{0} H e^{-x^{2}}\right]\right) .
$$

And therefore

$$
F_{\text {line }}^{\text {face }}=\frac{\pi R^{2}}{d^{2}} S_{0} G^{\text {face }}
$$

\subsection{Side-on flux}

For the Side-on view, radiation crossing the cylinder travels a different path depending on its distance from the center of the circle (see figure 2b). Defining an azimuthal angle $\phi$ (figure 2b), a ray crossing the cylinder travels a length $2 R \sin \phi$ inside the plasma. However, in this case, radiation does not leave the plasma perpendicular to the surface, but with an angle $\frac{\pi}{2}-\phi$, as shown in figure 2. Therefore the differential projected surface area seen by the detector is $H R \sin \phi d \phi$, so the differential flux for the Side-on orientation at a given azimuthal angle is:

$$
d F^{s i d e}=S_{0}\left(1-e^{-2 \kappa R \sin \phi}\right) \sin \phi \frac{H R d \phi}{d^{2}}
$$

Therefore, the flux integrated over the surface is

$$
F^{\text {side }}=\frac{H R}{d^{2}} S_{0} \int_{0}^{\pi}\left(1-e^{-2 \kappa R \sin \phi}\right) \sin \phi d \phi .
$$

This equation can be written explicitly by solving the integral.

$$
F^{\text {side }}=\frac{2 H R}{d^{2}} S_{0}\left[1-\frac{\pi}{2}\left(L_{-1}(2 \kappa R)-I_{1}(2 \kappa R)\right)\right]
$$

where $L_{n}$ is the modified Struve function of order $n$, and $I_{n}$ is the $n$-th order modified Bessel function of the first kind [55]. It is interesting to note here that 
in the optically thin limit $(\kappa R \rightarrow 0)$ the factor in square brackets in equation 16 reduces to $\left[1-\exp \left(-\frac{\pi}{2} \kappa R\right)\right]$. This is equivalent to assuming that all photons travel the average path length before leaving the plasma (the mean chord for Side-on view is $\pi R / 2$ ). However, this is not valid when the conditions are not entirely optically thin.

As it is difficult to work with equation 16 given the complicated functions inside the brackets, we approximate the exact solution by a function of the form $(1-\exp [-a \kappa R])$, by choosing a value of $a$ such that it minimizes the differences with the exact solution over a wide range of $\kappa R$ values. We chose a maximum value of $\kappa R$ of 10 to ensure convergence of the calculations. The optimal value of $a$ was found to be 1.45 . Using this approximation, there is less than $5 \%$ error for all values of $\kappa R$ in this range. The difference between both functions decreases as $\kappa R$ increases and both functions tend asymptotically to 1 . This difference becomes less than $1 \%$ for $\kappa R \sim 5$ and for $\kappa R=10$ its value is $\sim 0.25 \%$. Both our approximation and the exact solution are shown in figure 3

We recast the solution for the Side-on flux as

$$
F^{\text {side }} \sim \frac{2 H R}{d^{2}} \cdot S_{0}\left(1-e^{-1.45 \kappa R}\right) .
$$

and, in a similar way to the Face-on view, the frequency-integrated line flux is

$$
F_{\text {line }}^{\text {side }}=\frac{2 H R}{d^{2}} S_{0} \int_{-\infty}^{+\infty} d x\left(1-\exp \left[-1.45 \kappa_{0} R e^{-x^{2}}\right]\right) .
$$

This notation can be simplified in the same way as for the Face-on orientation, defining $G^{\text {side }}$ as the integral over frequencies for Side-on view

$$
G^{\text {side }}=\int_{-\infty}^{+\infty} d x\left(1-\exp \left[-1.45 \kappa_{0} R e^{-x^{2}}\right]\right) .
$$

Therefore

$$
F_{\text {line }}^{\text {side }}=\frac{2 H R}{d^{2}} S_{0} G^{\text {side }}
$$

\section{3. $w / y$ ratio}

In this section, we specify the flux corresponding to a particular line ( $w$ or $y$ ) by denoting $w$ or $y$ as a subscript. From now on, unless specified otherwise, 


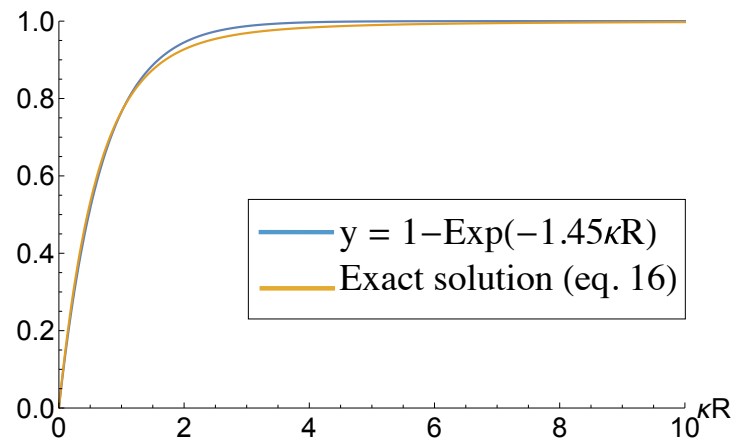

Figure 3: Comparison between the brackets in equation 16 (orange) and $1-e^{-1.45 \kappa R}$ (blue).

we work with frequency-integrated line fluxes (equations 13 and 20 , and drop the explicit subscript line. We also drop the subscript 0 for the source function, as we always refer to its value at line center for each line.

For the Face-on orientation, if the $w$ line flux is divided by the flux of the $y$ line, we obtain

$$
\left.\frac{F_{w}}{F_{y}}\right|_{\text {face }}=\frac{S_{w} G_{w}^{\text {face }}}{S_{y} G_{y}^{\text {face }}} .
$$

A similar expression is found for the Side-on view, substituting the $G$ integral,

$$
\left.\frac{F_{w}}{F_{y}}\right|_{\text {side }}=\frac{S_{w} G_{w}^{\text {side }}}{S_{y} G_{y}^{\text {side }}}
$$

Given that the source functions of both lines are independent of the direction of emission for uniform plasmas (they are a function of the plasma properties), if the $w / y$ ratios for both views are divided, the result depends only on the $G$ integrals for both lines in both views.

$$
\frac{F_{w} /\left.F_{y}\right|_{\text {face }}}{F_{w} /\left.F_{y}\right|_{\text {side }}}=\frac{G_{w}^{\text {face }}}{G_{w}^{\text {side }}} \cdot \frac{G_{y}^{\text {side }}}{G_{y}^{\text {face }}} .
$$

\section{Aspect ratio}

In most experiments, it is not possible to obtain direct measurements of the size of the plasma and the spectral flux in both directions at the same time. However, the fact that the $w$ line is optically thick allows us to obtain information about the plasma geometry just from the ratio of the spectral fluxes 
in two directions, without explicitly measuring the plasma size. In particular, we calculate the aspect ratio $\beta=H / R$. If the $w$ line fluxes for both views are divided, we obtain

$$
\frac{\left.F_{w}\right|_{\text {face }}}{\left.F_{w}\right|_{\text {side }}}=\frac{\pi R}{2 H} \frac{G_{w}^{\text {face }}}{G_{w}^{\text {side }}} .
$$

In the limit of high values of the optical depth, the ratio of the $G$ functions can be simplified. We use L'Hôpital's rule once

$$
\frac{G_{w}^{f a c e}}{G_{w}^{\text {side }}} \sim \lim _{\kappa_{0} \rightarrow \infty} \frac{H}{1.45 R} \frac{\int_{-\infty}^{+\infty} d x \exp \left[-\kappa_{0} H e^{-x^{2}}\right] e^{-x^{2}}}{\int_{-\infty}^{+\infty} d x \exp \left[-1.45 \kappa_{0} R e^{-x^{2}}\right] e^{-x^{2}}},
$$

and then Holstein's approximation for the resulting integrals [56], thus obtaining

$$
\frac{G_{w}^{\text {face }}}{G_{w}^{\text {side }}} \sim \sqrt{\frac{\ln \left(1.45 \kappa_{w} R\right)}{\ln \left(\beta \kappa_{w} R\right)}}=\sqrt{\frac{\ln \left(1.45 \cdot A \tau_{0}\right)}{\ln \left(A \beta \tau_{0}\right)}},
$$

where $\tau_{0}=\kappa_{y} R$. The factor $A=\kappa_{w} / \kappa_{y}$ is the ratio of the opacity of the $w$ and the $y$ lines, given by the ratio of the oscillator strengths of the transitions and therefore fixed for each element. It can be adjusted for the element of consideration, and it is independent of temperature and density. We introduce this factor as it is easier to work with the optical depth of the $y$ line, than the $w$ line. We show the dependence of the ratio $G_{w}^{\text {face }} / G_{w}^{\text {side }}$ with the optical depth of the $y$ line for different aspect ratios in figure 4 .

From equations 24 and 26, we can now obtain the following expression for the aspect ratio $\beta=H / R$

$$
\beta=\frac{\pi}{2} \cdot \frac{\left.F_{w}\right|_{\text {side }}}{\left.F_{w}\right|_{\text {face }}} \sqrt{\frac{\ln \left(1.45 \cdot A \tau_{0}\right)}{\ln \left(A \beta \tau_{0}\right)}}
$$

And therefore, equation 23 can be now written explicitly as

$$
\frac{F_{w} /\left.F_{y}\right|_{\text {face }}}{F_{w} /\left.F_{y}\right|_{\text {side }}}=\sqrt{\frac{\ln \left(1.45 \cdot A \tau_{0}\right)}{\ln \left(A \beta \tau_{0}\right)}} \cdot \frac{\int_{-\infty}^{+\infty} d x\left(1-\exp \left[-1.45 \tau_{0} e^{-x^{2}}\right]\right)}{\int_{-\infty}^{+\infty} d x\left(1-\exp \left[-\tau_{0} e^{-x^{2}} \beta\right]\right)},
$$

where we have kept the notation $\tau_{0}=\kappa_{y} R$ to indicate that, although $\kappa_{y}$ and $R$ cannot be calculated separately, their product is an independent variable in equations 27 and 28 . Physically, $\tau_{0}$ corresponds to the optical depth of a $y$ 


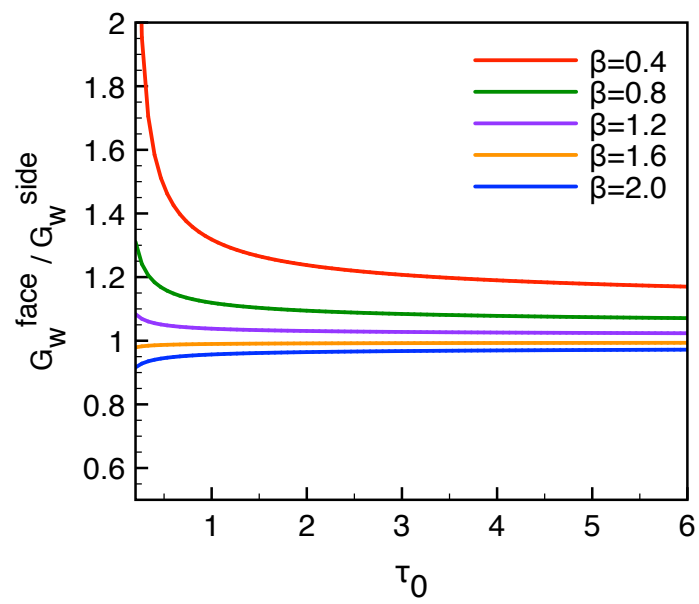

Figure 4: $G_{w}^{f a c e} / G_{w}^{\text {side }}$ ratio as a function of the optical depth of the $y$ line for a variety of aspect ratios. For the values considered in this document ( $\tau_{0}$ of the order of a few), this value is not strongly dependent on $\tau_{0}$, except at low aspect ratios.

line photon (at line center) travelling a distance equivalent to the radius of the cylinder, $R$.

Equations 27 and 28 can now be solved together, thus obtaining $\beta$ and $\tau_{0}$.

\section{Density calculation}

The optical depth parameter $\tau_{0}$ is related to the ion density of the plasma by

$$
\tau_{0}=n_{i} F \sigma_{0} R,
$$

where $n_{i}$ is the number density of ions, $F$ is the fraction of ions in the ground state of the transition (which, for the case of the $y$ line is the He-like ground state $\left.1 s^{2}{ }^{1} S_{0}\right)$ and $\sigma_{0}$ is the cross section for photon absorption at line center. For a line profile $\phi(x)$, the cross-section is

$$
\sigma_{0}=\frac{\pi e^{2}}{m_{e} c} f \phi(0),
$$

where $f$ is the oscillator strength of the transition, in this case the $y$ line. The 
ion density $n_{i}$ can be related to the total number of atoms in the plasma, $N$ as

$$
n_{i}=\frac{N}{V}=\frac{N}{\pi \beta R^{3}} .
$$

The quantity $N$, in turn, can be expressed as the initial mass of the target divided by the atomic mass, $N=M / m_{a}$ so that

$$
n_{i}=\frac{M}{m_{a} \pi \beta R^{3}} .
$$

Combining equations 29 and 32 , the following expression for the ion density is obtained

$$
n_{i}=\left(\frac{m_{a} \pi \beta}{M}\right)^{1 / 2}\left(\frac{\tau_{0}}{F \sigma_{0}}\right)^{3 / 2} .
$$

Equation 33 allows us to calculate the ion density of a plasma from its aspect ratio $\beta$ and the optical depth of the $y$ line at line center $\tau_{0}$. As we have shown in equations 27 and 28 , these two parameters can be calculated from the ratio of fluxes between the $w$ and the $y$ line for Face-on and Side-on views.

\section{A particular example}

To illustrate this method, we have built a computational model using the radiation transport code CRETIN. The characteristics of this model have been motivated by recent experiments at the OMEGA Laser Facility [57] which aim to benchmark the optical depth effects on the He $\alpha$ complex of cylindrical plasmas [58. Our model consists of a titanium plasma cylinder, with a radius $R=$ $200 \mu \mathrm{m}$ and an aspect ratio $\beta=0.8$, thus fixing the height to $H=160 \mu \mathrm{m}$. The temperature of the plasma is $1500 \mathrm{eV}$ and the plasma is in steady state. We fixed the ion density to be $n_{i}=2.0 \times 10^{19} \mathrm{~cm}^{-3}$. The total number of ions is then $N=V n_{i}=4.02 \times 10^{14}$. The Side-on and Face-on spectra obtained from the simulation are shown in figure 5a, where the positions of the $w$ and $y$ lines are indicated.

As the temperature of the cylinder is known in the simulation (in practice it can be determined self-consistently from spectroscopic measurements, as discussed in section 1), the Li-like structure can be modeled and subtracted from 


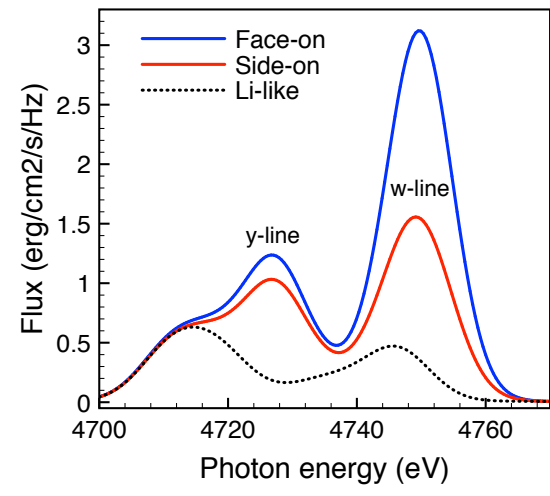

(a)

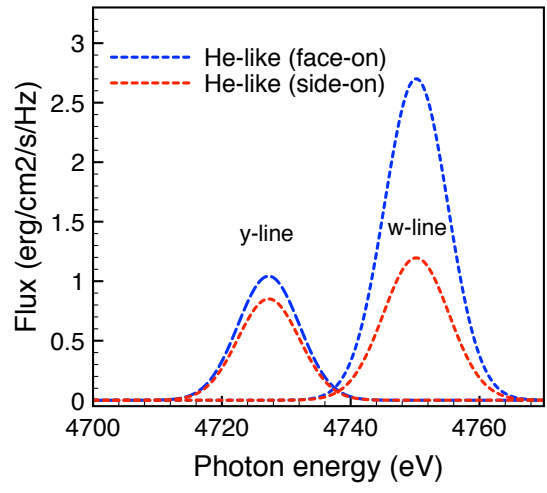

(b)

Figure 5: Spectra of the He-like complex for the example case. These correspond to the Face-on and Side-on flux of a Ti cylinder with an aspect ratio $\beta=0.8$ an ion density of $n_{i}=2 \times 10^{19} \mathrm{~cm}^{-3}$ at $T=1500 \mathrm{eV}$. Figure $5 \mathrm{a}$ shows the total flux including He-like and Li-like satellites. The Li-like contribution is shown for both cases as the dotted lines. In figure $5 \mathrm{~b}$ the satellite contribution has been subtracted and the He-like contribution is shown. We find only flux from the $w$ and $y$ lines, which can be fitted to two Gaussians to apply to process described in this document.

the data. This can be done because the Li-like satellite flux is optically thin and therefore, its spectral distribution is density-independent, and its magnitude is independent of the viewing orientation in the conditions of interest. The Li-like structure is shown as the black dotted line in figure 5a. We then obtain the He-like flux for each view, from which the $w$ and $y$ lines can be extracted. The He-like lines are shown in figure $5 b$,

In this particular example, the flux ratios obtained are:

$$
\begin{aligned}
& \frac{\left.F_{w}\right|_{\text {face }}}{\left.F_{w}\right|_{\text {side }}}=2.21, \\
& \frac{\left.F_{w}\right|_{\text {face }}}{\left.F_{y}\right|_{\text {face }}}=2.68, \\
& \frac{\left.F_{w}\right|_{\text {side }}}{\left.F_{y}\right|_{\text {side }}}=1.50 .
\end{aligned}
$$


We now solve equations 27 and 28 self-consistently with an iterative process. The results are $\beta=0.80$ and $\tau_{0}=0.61$.

From the plasma temperature $(1500 \mathrm{eV})$, we can calculate the cross section for photon scattering at the center of the $y$ line, obtaining $\sigma_{0}=1.70 \times 10^{-18} \mathrm{~cm}^{2}$. The fraction $F$, of ions in the He-like ground, that is, the product of the He-like ionic fraction and the fractional ground level population, can be estimated from the known temperature and calculations. Its value is 0.89 at this temperature, and therefore, the ion density calculated from equation 33 is

$$
n_{i}=2.0 \times 10^{19} \mathrm{~cm}^{-3} \text {. }
$$

In a real experiment, there will be experimental errors in the flux of the $w$ and $y$ lines in both directions, which will affect the measurement of the $w / y$ ratio. In addition, as we can see in figure $5 \mathrm{a}$, the apparent flux of the $\mathrm{He}$ lines has an important contribution from the Li-like satellites that needs to be removed with a model, introducing the potential for additional errors in the line flux. These sources of error will cause the measured $w / y$ ratios to differ from the theoretical ones, and will lead to an error on the calculated ion density. To study the sensitivity of this method to these errors, we allowed the flux from each line in both views to vary randomly within a $10 \%$ of the value from the simulations. This relatively standard error is estimated assuming that the temperature of the plasma is known from other spectroscopic measurements, and that the plasma is uniform. In case the temperature is not known, or there are non-uniformities in the plasma, the shape of the Li-like satellites will have a greater uncertainty and therefore, the error will be more important (there would be additional problems, as the cross-section for photon absorption also depends on the temperature). With this error included in the calculation, the ion density obtained is

$$
n_{i}=2.0 \pm 0.4 \times 10^{19} \mathrm{~cm}^{-3}
$$

As the simulation had a fixed ion density value of $n_{i}=2.0 \times 10^{19} \mathrm{~cm}^{-3}$, there is reasonable agreement with the obtained result. 


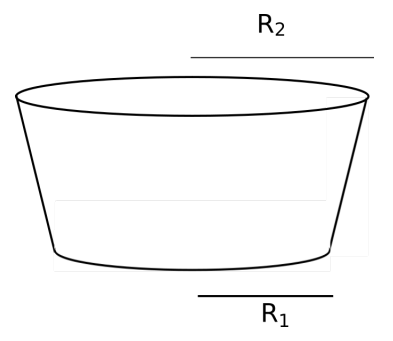

(a)

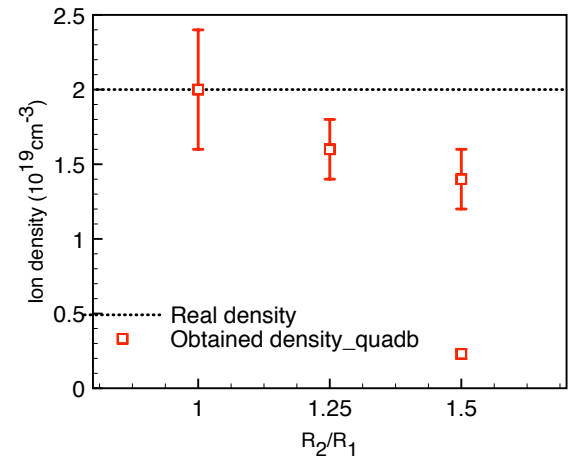

(b)

Figure 6: 6a Frustum geometry. 6b Ion density obtained applying this method to CRETIN simulations for a frustum as a function of the ratio between the radii of its bases. The point with $R_{2} / R_{1}=1$ corresponds to the cylindrical case shown in section 5

\section{Conclusions and future work}

We have introduced a novel method to obtain the ion density in a cylindrical HED plasma. This method only requires spectroscopic measurements of the He $\alpha$ region of the spectrum and can be complemented with different spectroscopic measurements of the plasma temperature. This is a general method for HED cylindrical plasmas. In particular, it is applicable to dot spectroscopy experiments in ICF conditions to diagnose the plasma inside the hohlraum, where the other commonly used diagnostics are difficult to yield.

The dependence of this method on the geometry of the plasma being cylindrical, although important, is not critical. If the plasma expands in solid angle into a frustum (figure 6a), therefore losing its cylindrical geometry, then the results obtained with this method start differing from the real ion density. The difference with the real value increases with the ratio $R_{2} / R_{1}$, where $R_{1}$ and $R_{2}$ are the radii of the minor and major bases of the frustum respectively. CRETIN simulations show that for values of this ratio up to 1.50 , the density obtained with this method is in agreement with the correct value within a factor of 2 , as figure 6 shows. Hence for small deviations of the cylindrical geometry this method can still be applied. 
Additional experiments at the OMEGA Laser Facility using mid-Z dots to benchmark the optical depth effects on the spectra as a function of the plasma geometry will provide an experimental platform to benchmark this method [58] with a multi-species plasma. We believe that this method could be used in ICF related Dot Spectroscopy experiments and HED plasmas.

\section{Acknowledgements}

G.P.-C., S. J. R. and J. S. W. gratefully acknowledge support from LLNL under grant number B617350.

This work was performed under the auspices of the U.S. Department of Energy by Lawrence Livermore National Laboratory under Contract DE-AC5207NA27344

\section{References}

[1] L. Heroux, A spectroscopic determination of electron temperature in a high temperature plasma, Proc. Phys. Soc. 83 (1). doi:10.1088/0370-1328/ $83 / 1 / 315$

[2] K. P. Dere, H. E. Mason, K. G. Widing, A. K. Bhatia, XUV electron density diagnostics for solar flares, Astrophys. J. Suppl. Ser. 40. doi: $10.1086 / 190590$

[3] J. Kilkeeny, R. Lee, M. Key, J. Lunney, X-ray spectroscopic diagnosis of laser-produced plasmas, with emphasis on line broadening, Phys. Rev. 22 (6). doi:10.1103/physreva.22.2746

[4] J. Apruzese, K. Whitney, J. Davis, P. Kepple, K-shell line ratios and powers for diagnosing cylindrical plasmas of neon, aluminum, argon, and titanium, J. Quant. Spectrosc. Radiat. Transfer 57 (1). doi:https://doi.org/10. 1016/S0022-4073(96)00097-0. 
[5] J. Apruzese, D. Duston, J. Davis, K-shell aluminum resonance line ratios for plasma diagnosis using spot spectroscopy, J. Quant. Spectrosc. Radiat. Transfer 36 (4). doi:https://doi.org/10.1016/0022-4073(86) 90057-9

[6] R. Marjoribanks, M. Richardson, P. Jaanimagi, R. Epstein, Electrontemperature measurement in laser-produced plasmas by the ratio of isoelectronic line intensities, Phys. Rev. 46 (4). doi:10.1103/physreva.46. r1747.

[7] C. J. Keane, B. A. Hammel, D. R. Kania, J. D. Kilkenny, R. W. Lee, A. L. Osterheld, L. J. Suter, R. C. Mancini, C. F. Hooper, N. D. Delamater, X-ray spectroscopy of high-energy density Inertial Confinement Fusion plasmas, Phys. Fluids B: Plasma Phys. 5 (9). doi:10.1063/1.860964.

[8] C. A. Back, D. H. Kalantar, R. L. Kauffman, R. W. Lee, B. K. MacGowan, D. S. Montgomery, L. V. Powers, T. D. Shepard, G. F. Stone, L. J. Suter, Measurements of electron temperature by spectroscopy in hohlraum targets, Phys. Rev. Lett. 77 (21). doi:10.1103/PhysRevLett.77.4350.

[9] T. D. Shepard, C. A. Back, D. H. Kalantar, R. L. Kauffman, C. J. Keane, D. E. Klem, B. F. Lasinski, B. J. MacGowan, L. V. Powers, L. J. Suter, R. E. Turner, B. H. Failor, W. W. Hsing, Isoelectronic x-ray spectroscopy to determine electron temperatures in long-scale-length Inertial-ConfinementFusion plasmas, Phys. Rev. E 53 (5). doi:10.1103/PhysRevE.53.5291.

[10] M. A. Barrios, D. A. Liedahl, M. B. Schneider, O. Jones, G. V. Brown, S. P. Regan, K. B. Fournier, A. S. Moore, J. S. Ross, O. Landen, R. L. Kauffman, A. Nikroo, J. Kroll, J. Jaquez, H. Huang, S. B. Hansen, D. A. Callahan, D. E. Hinkel, D. Bradley, J. D. Moody, Electron temperature measurements inside the ablating plasma of gas-filled hohlraums at the National Ignition Facility, Phys. Plasmas 23 (5). doi:10.1063/1.4948276.

[11] M. A. Barrios, J. D. Moody, L. J. Suter, M. Sherlock, H. Chen, W. Farmer, J. Jaquez, O. Jones, R. L. Kauffman, J. D. Kilkenny, J. Kroll, O. L. Landen, 
D. A. Liedahl, S. A. Maclaren, N. B. Meezan, A. Nikroo, M. B. Schneider, D. B. Thorn, K. Widmann, G. Pérez-Callejo, Developing an experimental basis for understanding transport in NIF hohlraum plasmas, Phys. Rev. Lett. 121. doi:10.1103/PhysRevLett.121.095002.

[12] J. Ashkenazy, R. Kipper, M. Caner, Spectroscopic measurements of electron density of capillary plasma based on stark broadening of hydrogen lines, Phys. Rev. A 43. doi:10.1103/PhysRevA.43.5568

[13] C. A. Morgan, H. R. Griem, R. C. Elton, Spectroscopic measurements of electron density and temperature in polyacetal-capillary-discharge plasmas, Phys. Rev. E 49. doi:10.1103/PhysRevE.49.2282.

[14] M. Ivković, S. Jovićević, N. Konjević, Low electron density diagnostics: development of optical emission spectroscopic techniques and some applications to microwave induced plasmas, Spectrochim. Acta, Part B 59 (5). doi:https://doi.org/10.1016/j.sab.2004.02.005.

[15] G. J. Bastiaans, R. A. Mangold, The calculation of electron density and temperature in Ar spectroscopic plasmas from continuum and line spectra, Spectrochim. Acta, Part B 40 (7). doi:https://doi.org/10.1016/ 0584-8547(85)80059-8.

[16] E. A. Den Hartog, T. R. O'Brian, J. E. Lawler, Electron temperature and density diagnostics in a helium glow discharge, Phys. Rev. Lett. 62. doi:10.1103/PhysRevLett.62.1500

[17] A. H. Gabriel, C. Jordan, Interpretation of solar helium-like ion line intensities, Mon. Not. R. Astron. Soc. 145 (2). doi:10.1093/mnras/145.2.241

[18] A. H. Gabriel, Dielectronic satellite spectra for highly-charged helium-like ion lines, Mon. Not. R. Astron. Soc. 160. doi:10.1093/mnras/160.1.99.

[19] G. A. Doschek, J. F. Meekins, The helium-like calcium, silicon, and sulfur lines during the decay of a large flare, Sol. Phys. 13 (1). doi:10.1007/ BF00963954, 
[20] G. R. Blumenthal, G. W. F. Drake, W. H. Tucker, Ratio of line intensities in helium-like ions as a density indicator., Astrophys. J. 172. doi:10.1086/ 151340 .

[21] L. W. Acton, R. C. Catura, A. J. Meyerott, C. J. Wolfson, J. L. Culhane, Coronal survey in X-rays of O VIII and Ne IX, Sol. Phys. 26 (1). doi: 10.1007/BF00155119

[22] F. P. Keenan, S. S. Tayal, A. E. Kingston, Theoretical NeIX line ratios compared to solar observations, Sol. Phys. 94 (1). doi:10.1007/BF00154809.

[23] F. P. Keenan, W. A. Feibelman, K. A. Berrington, Improved calculations for the C III 1907,1909 and Si III 1883,1892 electron density sensitive emission-line ratios, and a comparison with IUE observations, Astrophys. J. 389. doi:10.1086/171220.

[24] Porquet, D., Mewe, R., Dubau, J., Raassen, A. J. J., Kaastra, J. S., Line ratios for helium-like ions: Applications to collision-dominated plasmas, Astron. Astrophys. 376 (3). doi:10.1051/0004-6361:20010959.

[25] J.-U. Ness, R. Mewe, J. H. M. M. Schmitt, A. J. J. Raassen, D. Porquet, J. S. Kaastra, R. L. J. van der Meer, V. Burwitz, P. Predehl, Heliumlike triplet density diagnostics - Applications to CHANDRA-LETGS Xray observations of Capella and Procyon, Astron. Astrophys. 367 (1). doi: 10.1051/0004-6361:20000419

[26] J.-U. Ness, J. H. M. M. Schmitt, V. Burwitz, R. Mewe, A. J. J. Raassen, R. L. J. van der Meer, P. Predehl, A. C. Brinkman, Coronal density diagnostics with Helium-like triplets: CHANDRA-LETGS observations of Algol, Capella, Procyon, $\epsilon$ Eri, $\alpha$ Cen A\&B, UX Ari, AD Leo, YY Gem, and HR 1099, Astron. Astrophys. 394 (3). doi:10.1051/0004-6361:20021146.

[27] D. Porquet, J. Dubau, N. Grosso, He-like ions as practical astrophysical plasma diagnostics: From stellar coronae to active galactic nuclei, Space Sci. Rev. 157 (1). doi:10.1007/s11214-010-9731-2. 
[28] A. V. Vinogradov, I. Y. Skobelev, E. A. Yukov, Determination of plasma density from spectra of heliumlike ions, Sov. J. Quantum Electron. 5 (6). doi:10.1070/QE1975v005n06ABEH011298.

[29] V. P. Shevelko, I. Y. Skobelev, A. V. Vinogradov, Density-dependent lines of one- and two-electron ions in diagnostics of laboratory plasma. I. The rates of collision relaxation of excited levels, Phys. Scr. 16 (3-4). doi: 10.1088/0031-8949/16/3-4/010

[30] I. Y. Skobelev, A. V. Vinogradov, E. A. Yukov, Density-dependent lines of one- and two-electron ions in diagnostics of laboratory plasma. II. Intensity line ratios of hydrogenlike, heliumlike and oxygenlike multicharged ions, Phys. Scr. 18 (2). doi:doi:10.1088/0031-8949/18/2/003.

[31] V. A. Boiko, A. Y. Chugunov, A. Y. Faenov, S. A. Pikuz, I. Y. Skobelev, A. V. Vinogradov, E. A. Yukov, Anomalous intensity ratios of the resonance to intercombination lines of He-like ions in Nd- and $\mathrm{CO} 2$-laser-produced plasmas, J. Phys. B: At. Mol. Phys. 12 (2). doi:10.1088/0022-3700/12/ $2 / 012$

[32] V. Boiko, A. Faenov, S. Pikuz, X-ray spectroscopy of multiply-charged ions from laser plasmas, J. Quant. Spectrosc. Radiat. Transfer 19 (1). doi: https://doi.org/10.1016/0022-4073(78)90038-9.

[33] D. Duston, J. Davis, Line emission from hot, dense, aluminum plasmas, Phys. Rev. A 21. doi:10.1103/PhysRevA.21.1664.

[34] M. Chaker, J. C. Kieffer, J. P. Matte, H. Ppin, P. Audebert, P. Maine, D. Strickland, P. Bado, G. Mourou, Interaction of a 1 psec laser pulse with solid matter, Phys. Fluids B: Plasma Phys. 3 (1). doi:10.1063/1.859934

[35] H. Fiedorowicz, A. Bartnik, Z. Patron, P. Parys, X-ray emission from laserirradiated gas puff targets, Appl. Phys. Lett. 62 (22). doi:10.1063/1. 109232 . 
[36] J. G. Doyle, J. L. Schwob, Intercombination to resonance line intensity ratio for He-like oxygen and carbon ions in TFR tokamak plasmas, J. Phys. B: At. Mol. Phys. 15 (5) (1982) 813. doi:10.1088/0022-3700/15/5/023

[37] M. Bitter, K. W. Hill, S. v. Goeler, W. Stodiek, P. Beiersdorfer, J. E. Rice, A. Ince-Cushman, Measurements of the intercombination and forbidden lines from the spectra of helium-like ions in tokamaks and electron beam ion traps, Can. J. Phys. 86 (1). doi:10.1139/p07-138.

[38] A. K. Bhatia, S. O. Kastner, The optically thick Fe XVII spectrum: Xray, extreme-ultraviolet, and forbidden line ratios, Astrophys. J. 516 (1). doi:10.1086/307085

[39] A. K. Bhatia, J. L. R. Saba, Resonance scattering of Fe XVII X-ray and extreme-ultraviolet lines, Astrophys. J. 563 (1). doi:10.1086/323688.

[40] S. O. Kastner, A. K. Bhatia, Optically thin and thick Fe XV spectrum: Effect of self-absorption on the $284.16 \AA$ A resonance line, Astrophys. J. 553 (1). doi:10.1086/320649

[41] F. M. Kerr, S. J. Rose, J. S. Wark, F. P. Keenan, Enhancement of optically thick to thin line intensities in solar and stellar coronal plasmas through radiative transfer effects: an angularly resolved study, Astrophys. J. 613. doi:10.1086/425177

[42] F. Kerr, S. Rose, J. Wark, An analytic geometry-variant approach to line ratio enhancement above the optically thin limit, Astrophys. J. 629 (2). doi:10.1086/429881

[43] F. Kerr, A. Gouveia, O. Renner, S. Rose, H. Scott, J. Wark, Line radiation effects in laboratory and astrophysical plasmas, J. Quant. Spectrosc. Radiat. Transfer 99 (1). doi:10.1016/j.jqsrt.2005.05.029.

[44] H. A. Scott, CRETIN - a radiative transfer capability for laboratory plasmas, J. Quant. Spectrosc. Radiat. Transfer 71 (2). doi:https://doi.org/ 10.1016/S0022-4073(01)00109-1. 
[45] F. P. Keenan, J. G. Doyle, M. S. Madjarska, S. J. Rose, L. A. Bowler, J. Britton, L. McCrink, M. Mathioudakis, Intensity enhancement of O VI ultraviolet emission lines in solar spectra due to opacity, Astrophys. J. 784. doi:doi:10.1088/2041-8205/784/2/L39.

[46] P. Hatfield, Using line intensity ratios to determine the geometry of plasma in stars via their apparent areas, High Energy Density Phys. 6 (3). doi: https://doi.org/10.1016/j.hedp.2009.10.001.

[47] R. C. Mancini, J. E. Bailey, J. F. Hawley, T. Kallman, M. Witthoeft, S. J. Rose, H. Takabe, Accretion disk dynamics, photoionized plasmas, and stellar opacities, Phys. Plasmas 16 (4). doi:10.1063/1.3101819.

[48] O. S. Jones, L. J. Suter, H. A. Scott, M. A. Barrios, W. A. Farmer, S. B. Hansen, D. A. Liedahl, C. W. Mauche, A. S. Moore, M. D. Rosen, J. D. Salmonson, D. J. Strozzi, C. A. Thomas, D. P. Turnbull, Progress towards a more predictive model for hohlraum radiation drive and symmetry, Phys. Plasmas 24 (5). doi:10.1063/1.4982693.

[49] G. H. Miller, E. I. Moses, C. R. Wuest, The National Ignition Facility, Opt. Eng. 43. doi:10.1117/1.1814767

[50] S. Hansen, J. Bauche, C. Bauche-Arnoult, M. Gu, Hybrid atomic models for spectroscopic plasma diagnostics, High Energy Density Phys. 3 (1), Radiative Properties of Hot Dense Matter. doi:https://doi.org/10. 1016/j.hedp.2007.02.032.

[51] H. R. Griem, Plasma Spectroscopy, McGraw-Hill, 1964.

[52] H. R. Griem, Spectral Line Broadening by Plasmas, Academic Press, 1974.

[53] D. Salzmann, Atomic Physics in Hot Plasmas, Oxford University Press, 1998.

[54] D. Mihalas, Stellar Atmospheres - Second Edition, San Francisco: W. H. Freeman \& Company, 1978. 
[55] M. Abramowitz, I. Stegun, Handbook of Mathematical Functions: With Formulas, Graphs, and Mathematical Tables, Applied mathematics series, Dover Publications, 1965.

[56] T. Holstein, Imprisonment of resonance radiation in gases, Phys. Rev. 72. doi:10.1103/PhysRev.72.1212.

[57] T. Boehly, D. Brown, R. Craxton, R. Keck, J. Knauer, J. Kelly, T. Kessler, S. Kumpan, S. Loucks, S. Letzring, F. Marshall, R. McCrory, S. Morse, W. Seka, J. Soures, C. Verdon, Initial performance results of the OMEGA laser system, Opt. Commun. 133 (1) (1997) 495 - 506. doi:https://doi. org/10.1016/S0030-4018(96)00325-2.

[58] G. Pérez-Callejo, et al., in preparation. 